\title{
Sperm retrieval by conventional testicular sperm extraction for assisted reproduction in patients with Zinner syndrome
}

\author{
Gianmartin Cito ${ }^{1}$, Luca Gemma ${ }^{1}$, Claudia Giachini ${ }^{2}$, Elisabetta Micelli ${ }^{2}$, Andrea Cocci ${ }^{1}$, Rossella Fucci ${ }^{2}$, Rita Picone ${ }^{2}$, \\ Simone Sforza ', Gabriella Nesi ${ }^{3}$, Raffaella Santi ${ }^{3}$, Andrea Minervini' , Lorenzo Masieri ', Marco Carini ', Maria Elisabetta \\ Coccia ${ }^{2, *}$, Alessandro Natali ${ }^{1, *}$
}

'Department of Urology, Careggi Hospital, ${ }^{2}$ Assisted Reproductive Technology Centre, Careggi Hospital, and ${ }^{3}$ Pathology Section, Department of Health Sciences, University of Florence, Florence, Italy

We present data from three Caucasian men with Zinner syndrome who attended our center for the treatment of primary couple's infertility. Each patient was scheduled for conventional testicular sperm extraction (CTESE) and cryopreservation. Sperm analysis confirmed absolute azoospermia. Patient 1 had right and left testis volumes of $24 \mathrm{~mL}$ and $23 \mathrm{~mL}$, respectively; left seminal vesicle (SV) agenesis, severe right SV hypotrophy with right renal agenesis. Follicle-stimulating hormone (FSH) was $3.2 \mathrm{lU} / \mathrm{L}$. Patient 2 exhibited right and left testis volumes of 18 $\mathrm{mL}$ and $16 \mathrm{~mL}$, respectively; a left SV cyst of $32 \times 28 \mathrm{~mm}$, ipsilateral kidney absence, and right SV agenesis. FSH was $2.8 \mathrm{IU} / \mathrm{L}$. Patient 3 showed a testicular volume of $10 \mathrm{~mL}$ bilaterally, a $65 \times 46 \mathrm{~mm}$ left SV cyst, right SV enlargement, and left kidney agenesis. FSH was $32.0 \mathrm{lU} / \mathrm{L}$. Sperm retrieval was successful in all patients. Nevertheless, cTESE should be performed on the day of oocyte retrieval.

Keywords: Azoospermia; Infertility; Testicular sperm retrieval; Wolffian duct anomalies; Zinner syndrome

\section{Introduction}

Zinner syndrome (ZS) is one of the rarest congenital anomalies of the urogenital tract. Due to its lack of symptoms, it is usually discovered and diagnosed in the second to fourth decades of life [1]. ZS can be considered a variant of the Wolffian duct anomalies, with a classic triad of seminal vesicle cyst, ipsilateral renal agenesis, and male infertility due to ejaculatory duct obstruction (EDO). The prevalence of

Received: April 19, 2020 - Revised: July 3, 2020 · Accepted: September 8, 2020 Corresponding author: Gianmartin Cito

Department of Urology, Careggi Hospital, University of Florence, Largo Brambilla, 3, 50134 Florence, Italy

Tel: +39-0557949203 Fax: +39-0557949203 E-mail: gianmartin.cito@gmail.com

*These authors contributed equally to this work.

This is an Open Access article distributed under the terms of the Creative Commons Attribution Non-Commercial License (http://creativecommons.org/licenses/by-nc/4.0/) which permits unrestricted non-commercial use, distribution, and reproduction in any medium, provided the original work is properly cited. cystic lesions in the pelvis with ipsilateral renal agenesis or dysplasia has been reported to be as high as $0.0046 \%$ [2]. Usually, this condition is also associated with other urogenital findings such as ureterocele, hypospadias, or abnormalities of the testes, epididymis, or adrenal glands $[3,4]$. The embryological origins of the kidneys and seminal vesicles are similar; the kidney is formed by the metanephric blastema, which is induced by the ureteral bud that originates from the dorsal aspect of the distal mesonephric duct. The mesonephric duct gives rise to most of the genital tract, including the epididymis, vas deferens, ejaculatory duct (ED), and seminal vesicles [5]. Thus, ZS can be considered the result of any malformation of the ureteral bud or mesonephric duct.

Patients with ZS are often asymptomatic, although aspecific signs may be present such as pelvic pain, painful ejaculation, urinary frequency, dysuria, urgency, constipation, recurrent urinary tract infections, or epididymitis [6]. As a consequence of the wide range of symptoms of this syndrome, the diagnosis of infertility can be de- 
layed, and ZS requires prompt management upon proper diagnosis. Semen evaluation is unlikely to reveal absolute azoospermia, but commonly reveals obstructive azoospermia (OA), although secretory damage may also be present due to a long-lasting obstruction to sperm outflow [6]. While the surgical removal of seminal vesicle cysts [7] or transurethral resection of the ED (TURED) may improve sperm quality [8], testicular fine needle aspiration, epididymal percutaneous sperm aspiration, and testicular sperm extraction (TESE) may be crucial to obtain viable spermatozoa and allow a chance of conception.

The aim of this work was to describe the outcomes of surgical sperm retrieval (SSR) in infertile patients with ZS who underwent conventional TESE (cTESE) for assisted reproduction.

\section{Case report}

We collected data from three Caucasian men with ZS who attended our assisted reproductive technology (ART) center for the treatment of primary couple's infertility. Written informed consent to use their data anonymously was obtained. In accordance with the 2010 World Health Organization guidelines [9], two semen analyses revealed absolute azoospermia, low ejaculate volume $(<1 \mathrm{~mL})$, and normal semen $\mathrm{pH}$ (Table 1) in all patients. The mean duration of infertility was $24 \pm 3$ months. No previous surgery, ongoing pathologies, or genetic abnormalities were reported. All patients were scheduled for multiple cTESE with cryopreservation.

Patient 1 was 27 years old. No history of cryptorchidism, delayed pubertal development, or scrotal-perineal discomfort was reported in his past medical records. His partner was 29 years old and did not report any gynecological diseases, except for a brief period of stress amenorrhea. Physical examination of the external genitalia detected a testicular volume of approximately $25 \mathrm{~mL}$ bilaterally, dilatation of the right vas deferens and epididymis, nonpalpable left vas deferens/ epididymis structures, and no clinical signs of varicocele bilaterally. Scrotal and transrectal ultrasound (US) examinations showed right and left testis volumes of $24 \mathrm{~mL}$ and $23 \mathrm{~mL}$, respectively; dilatation of the right caput epididymis, corpus, and cauda; and left seminal vesicle agenesis and right seminal vesicle severe hypotrophy. Transabdominal US revealed the absence of the right kidney as well as left nephroptosis. An abdominal contrast-enhanced computed tomography $(\mathrm{CT})$ scan confirmed these findings.

In this patient, the follicle-stimulating hormone (FSH) level was 3.2 $\mathrm{IU} / \mathrm{L}$, the luteinizing hormone $(\mathrm{LH})$ level was $5.2 \mathrm{IU} / \mathrm{L}$, the total testosterone (TT) level was $22.3 \mathrm{nmol} / \mathrm{L}$, the prolactin (PRL) level was 22 $\mathrm{ng} / \mathrm{mL}$, and the $17 \beta$-estradiol level was $0.12 \mathrm{nmol} / \mathrm{L}$. SSR was successful: the sperm concentration was $0.01 \times 10^{6} / \mathrm{mL}$, the sperm motility was $1 \%$, and five straws were cryopreserved. Evaluation of the testicular histology showed a pattern of normal spermatogenesis, thickening of tubular membranes and interstitial edema (Figure 1), and no germ cell neoplasia in situ (GCNIS).

Patient 2 was 31 years old with no past medical history. His partner was 30 years old and had a history of ovarian endometriosis. Genital examination revealed testicular volumes of approximately $20 \mathrm{~mL}$ on the right and $18 \mathrm{~mL}$ on the left side, mild dilatation of the left vas deferens and epididymis, and marked dilatation of the right vas deferens and epididymis structures. US showed right and left testis volumes of $18 \mathrm{~mL}$ and $16 \mathrm{~mL}$, respectively; a left seminal vesicle cyst with dimensions of $32 \times 28 \mathrm{~mm}$ and fluid content; right seminal vesicle agenesis; and left renal agenesis.

The patient's levels of FSH, LH, TT, PRL, and 17 $\beta$-estradiol were 2.8 $\mathrm{IU} / \mathrm{L}, 4.2 \mathrm{IU} / \mathrm{L}, 14.3 \mathrm{nmol} / \mathrm{L}, 21 \mathrm{ng} / \mathrm{mL}$, and $0.11 \mathrm{nmol} / \mathrm{L}$, respectively. SSR was successful: the sperm concentration was $0.01 \times 10^{6} / \mathrm{mL}$, the sperm motility was $1 \%$, and four straws were cryopreserved. Histological assessment showed a pattern of normal spermatogenesis, without findings indicative of GCNIS.

Patient 3 was 27 years old, and his partner was 26 years old. Persistent scrotal-perineal pain and dysuria during the last 6 months were reported. The andrological examination showed a testicular volume of approximately $10 \mathrm{~mL}$ bilaterally. The patient's levels of FSH, LH, TT, PRL, and 17 $\beta$-estradiol were $32.0 \mathrm{IU} / \mathrm{L}, 16.3 \mathrm{IU} / \mathrm{L}, 9.0$ $\mathrm{nmol} / \mathrm{L}, 21 \mathrm{ng} / \mathrm{mL}$, and $0.12 \mathrm{nmol} / \mathrm{L}$, respectively. US showed (1) an oval hypodense mass with dimensions of $65 \times 46 \mathrm{~mm}$ and fluid con-

Table 1. Comparison of seminal parameters among patients

\begin{tabular}{|c|c|c|c|c|}
\hline Variable & Patient 1 & Patient 2 & Patient 3 & Normal range \\
\hline Abstinence (day) & 3 & 3 & 5 & $2-7$ \\
\hline Color & Opalescent white & Opalescent white & Opalescent white & - \\
\hline Viscosity & Normal & Normal & Normal & - \\
\hline Liquefaction & Complete & Complete & Complete & - \\
\hline Semen volume (mL) & 0.2 & 0.3 & 0.6 & $>1.5$ \\
\hline Semen pH & 7.4 & 7.2 & 7.6 & $7.2-7.8$ \\
\hline Concentration (million/mL) & 0 & 0 & 0 & $>15$ \\
\hline Leukocyte & $<1$ million & $<1$ million & $<1$ million & $<1$ million \\
\hline
\end{tabular}




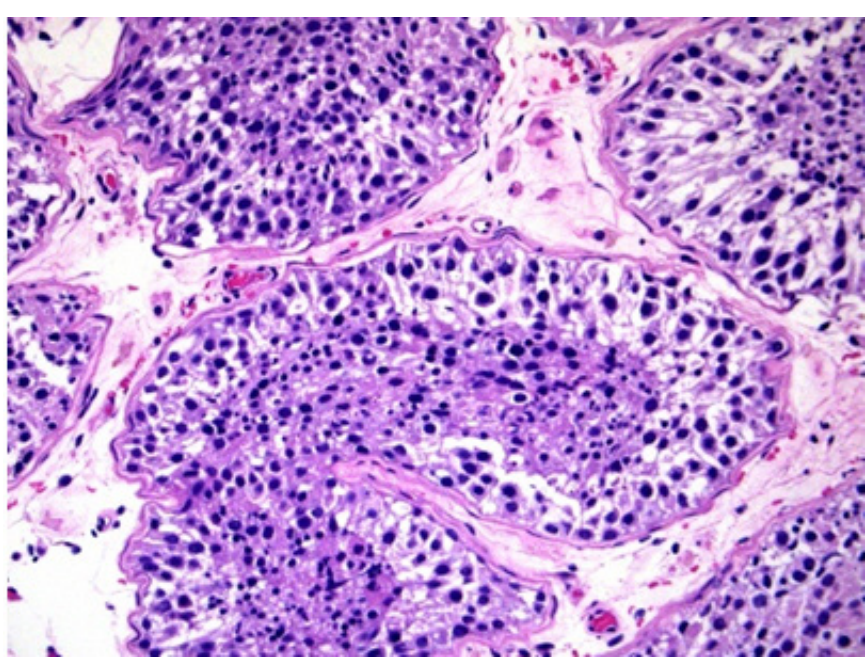

Figure 1. Cross-section of the seminiferous tubules with complete spermatogenesis surrounded by the tunica propria $(H \& E, \times 200)$.

tent that impacted the bladder and the left paramedian prostatic region and was compatible with a left seminal vesicle pseudocyst; and (2) enlargement of the right seminal vesicle. Abdominal contrast-enhanced CT confirmed these data and also revealed left kidney agenesis (Figure 2). The patient underwent left robot-assisted laparoscopic vesiculectomy (Figure 3). At the 12-month follow-up visit, no sperm were found in the semen analysis. Therefore, conventional bilateral TESE was proposed. SSR was successful: the concentration was $0.001 \times 10^{6} / \mathrm{mL}$, the sperm motility was $1 \%$, and three straws were cryopreserved. Histological assessment indicated hypospermatogenesis without GCNIS.

\section{Discussion}

ZS is a rare condition that is usually diagnosed in adulthood during a couple's infertility assessment [10]. The diagnosis is mainly based on imaging examinations that reveal the typical cystic dilatations of the seminal vesicle and ipsilateral renal agenesis [11-14]. In our series, two patients had conditions resembling the classic ZS variant, while 1 (patient 1) had right seminal vesicle severe hypotrophy and an absence of the ipsilateral kidney. Given the presence of the triad of unilateral renal agenesis, ipsilateral seminal vesicle obstruction, and ipsilateral EDO, this clinical presentation can be considered a rare type of embryological malformation, included as a pseudo-ZS variant.

ZS is often asymptomatic until the age of peak sexual and reproductive activity, usually after repeated failure to achieve natural conception. Incomplete EDO, which occurs in approximately $4 \%$ of these patients, can cause seminal abnormalities, which create difficulties in diagnosis [15]. In our study, in two patients, the diagnosis was made
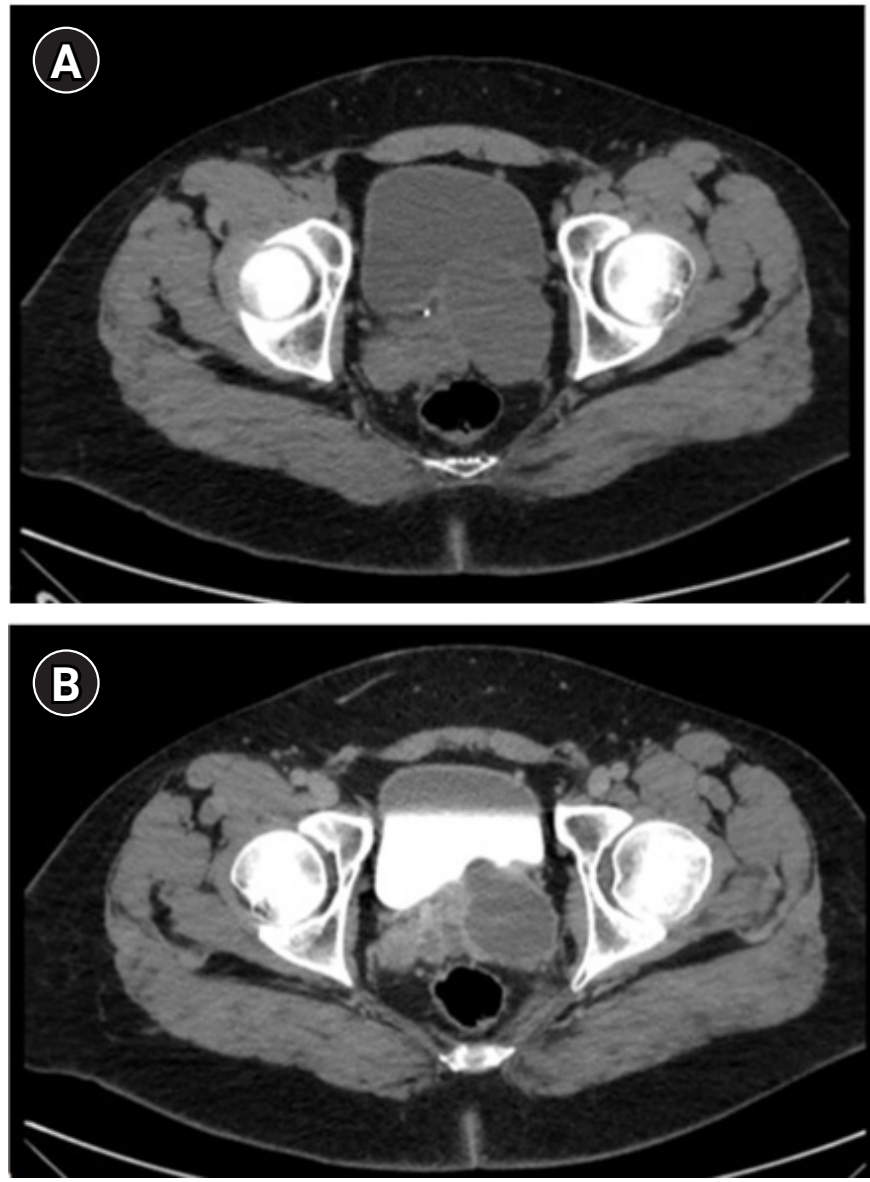

Figure 2. Abdominal contrast-enhanced computed tomography (CT) scan showing a left seminal vesicle pseudocyst. (A) Noncontrast CT. (B) CT urogram.



Figure 3. Robot-assisted laparoscopic vesiculectomy. A transversal incision of the peritoneum was performed, and the retrovesical region was sectioned using monopolar scissors and a bipolar Maryland dissector. A large cyst of the left seminal vesicle (SV) was isolated and liberated from the contiguous tissue. P, prostate; B, bladder. 
based on the occurrence of infertility without any urological complaints. The findings of absolute azoospermia via seminal analysis required further diagnostic investigations, including abdominal, scrotal, and transrectal US. Only one patient (patient 3) reported scrotal-perineal pain and dysuria that required further examination. Given the broad spectrum of symptoms, the diagnosis of ZS can be delayed, which can further compromise the patient's reproductive potential.

From a pathophysiological standpoint, ZS can be associated with seminal tract obstruction, and secretory damage may occur due to the presence of a long-lasting blockage to sperm outflow [6]. Although TURED represents the first-line treatment in patients with EDO to restore fertility and improve sperm quality, testicular fine-needle aspiration, epididymal percutaneous sperm aspiration, or TESE are effective options to obtain viable spermatozoa. In the literature, the semen improvement and paternity rates have been recorded across multiple studies as $38 \%$ to $60 \%$ and $22 \%$ to $31 \%$, respectively, after TURED [8]. Otherwise, SSR by testicular surgery in cases of OA usually has a success rate of around $90 \%-100 \%$ [16]. No patient described in this report underwent vesiculography and endoscopic procedures to enhance fertility. Robot-assisted laparoscopic vesiculectomy was performed in patient 3 to treat his clinical symptoms (chronic pelvic pain) due to the large seminal pseudocyst that affected the bladder. In that case, TESE was proposed at the 1 -year follow-up visit, in the absence of semen quality improvement. Then, patients 1 and 2, according to their preference, decided to undergo TESE, given that they had already started an ART program with their partners.

Long-lasting obstruction (that is, that lasts for several years) has been proposed to potentially cause germ cell failure in the spermatogenetic tubules [6]. The elevated concentrations of macrophages and sperm fragments in the distal epididymis may affect the sperm quality [17]. Moreover, according to previous authors, higher levels of reactive oxygen species and sperm DNA fragmentation could mediate the reproductive toxicity [18]. In our patients, the presence of high levels of polyunsaturated fatty acids on the sperm membrane; the low quantities of glutathione peroxidase, superoxide dismutase, and cytoplasm; and the presence of inactive, highly condensed chromatin could have led to a reduction in fertilization competence, motility, and viability, as well as a loss of DNA repair capacity [19].

When we proposed cTESE to our patients, we questioned whether it was appropriate to carry out the procedure to obtain fresh sperm (on the same day as oocyte retrieval/intracytoplasmic sperm injection [ICSI]) or to plan sperm cryopreservation. The question arose from awareness that patients with ZS exhibit an embryological defect resulting from the abnormal development of the mesonephric or Wolffian duct with abnormal development of the ipsilateral upper urinary tract. We were not certain whether these patients may also exhibit spermatogenetic failure. Thus, although SSR in cases of OA usually has nearly a $100 \%$ success rate [20], we assumed that cases of ZS may also involve damage to the germ line associated with impaired spermatogenesis, which is why we preferred to schedule cryopreservation anyway.

The ART outcomes of surgically retrieved fresh and cryopreserved sperm for assisted reproduction remain a topic of debate. High fertilization and pregnancy rates have been reported in in vitro fertilization (IVF)/ICSI procedures using fresh sperm obtained by TESE in both patients with $\mathrm{OA}$ and those with non-OA [21,22]. Usually, cryopreservation decreases the number of viable sperm by at least $50 \%$ [23]. Moreover, many cryopreserved testis specimens from men with non-OA have been found to have insufficient motile sperm after thawing. Thus, the fertilization rate has been described to be higher when fresh motile sperm were used than when frozen-thawed sperm were used. Furthermore, higher spontaneous abortion rates after implantation have also been seen when immotile spermatozoa were used. According to the experience of practitioners at our center, using fresh rather than cryopreserved sperm optimizes the ICSI outcomes, as these outcomes may be influenced by the effects of freezing and thawing on the sperm membrane.

Several authors have demonstrated similar IVF/ICSI outcomes for both sperm-handling methods [24,25]. The rupture of the cell membrane due to osmotic stress and the formation of ice crystals in freeze-thaw cycles could facilitate the release, dissolution, and integration of DNA once the sperm is injected into the oocyte cytoplasm, leading to higher fertilization rates $[24,26]$. Given that diagnostic testicular biopsy may be considered obsolete, it is crucial to establish preoperatively all of the possible predictive factors of successful SSR. Although a fresh procedure could maximize the potential of retrieving viable spermatozoa, cryopreservation before ICSI could represent the treatment of choice, as it could obviate the possibility of unexpected testicular failure on the day of the IVF procedure.

This is the first study to describe the outcomes of SSR in patients with ZS. In our case series, sperm recovery by cTESE was successful in all patients. Although the coexistence of a defect in the germ line cells can be supposed, the histological findings demonstrated that spermatogenesis was normal in these patients. As the normal transport of sperm through the ductal system is altered by a congenital defect in ZS patients, surgical extraction remains an excellent option for the management of $\mathrm{ZS}$.

In conclusion, in patients with ZS, it may be advisable to perform CTESE to obtain fresh sperm on the same day as oocyte retrieval. Sperm cryopreservation should be avoided, since the thawing procedure could further compromise the sperm quality. However, in the 
case of unsuccessful SR, oocyte freezing would avoid the nullification of controlled ovarian hyperstimulation and allow the use of the cryopreserved female gametes for a future IVF cycle via sperm donation. In this scenario, proven laboratory experience with oocyte thawing is key to successful ART outcomes.

\section{Conflict of interest}

No potential conflict of interest relevant to this article was reported.

\section{ORCID}

\section{Gianmartin Cito Luca Gemma}

https://orcid.org/0000-0001-7526-4025 https://orcid.org/0000-0001-6576-3218

\section{Author contributions}

Conceptualization, Formal analysis, Methodology, \& Data curation: $\mathrm{GC}, \mathrm{LG}, \mathrm{CG}, \mathrm{EM}, \mathrm{AC}, \mathrm{RF}, \mathrm{RP}, \mathrm{SS}, \mathrm{GN}$, RS. Project administration: AM, LM, MC. Writing-original drafting: GC, LG. Writing-review \& editing: MEC, AN.

\section{References}

1. Mehra S, Ranjan R, Garga UC. Zinner syndrome: a rare developmental anomaly of the mesonephric duct diagnosed on magnetic resonance imaging. Radiol Case Rep 2016;11:313-7.

2. Farooqui A, AlDhahir L, Mahfooz AB. Massive seminal vesicle cyst with ipsilateral renal agenesis: Zinner syndrome in a Saudi patient. Urol Ann 2018;10:333-5.

3. Pereira BJ, Sousa L, Azinhais P, Conceicao P, Borges R, Leao R, et al. Zinner's syndrome: an up-to-date review of the literature based on a clinical case. Andrologia 2009;41:322-30.

4. Tan Z, Li B, Zhang L, Han P, Huang H, Taylor A, et al. Classifying seminal vesicle cysts in the diagnosis and treatment of Zinner syndrome: a report of six cases and review of available literature. Andrologia 2020;52:e13397.

5. Slaoui A, Regragui S, Lasri A, Karmouni T, El Khader K, Koutani A, et al. Zinner's syndrome: report of two cases and review of the literature. Basic Clin Androl 2016;26:10.

6. Cito G, Sforza S, Gemma L, Cocci A, Di Maida F, Dabizzi S, et al. Infertility case presentation in Zinner syndrome: can a long-lasting seminal tract obstruction cause secretory testicular injury? Andrologia 2019;51:e13436.

7. Benyo M, Berczi C, Jozsa T, Csanadi G, Varga A, Flasko T. Fertility preservation in cases of laparoscopic treatment of seminal vesicle cysts. Cent European J Urol 2012;65:144-5.

8. Pace G, Galatioto GP, Guala L, Ranieri G, Vicentini C. Ejaculatory duct obstruction caused by a right giant seminal vesicle with an ipsilateral upper urinary tract agenesia: an embryologic malformation. Fertil Steril 2008;89:390-4.

9. Cooper TG, Noonan E, von Eckardstein S, Auger J, Baker HW, Behre HM, et al. World Health Organization reference values for human semen characteristics. Hum Reprod Update 2010;16:23145.

10. Aghaways I, Ahmed SM. Endourologic intervention for management of infertility in a man with Zinner syndrome resulting in a natural pregnancy. J Endourol Case Rep 2016;2:71-3.

11. Hergan B, Fellner FA, Akbari K. Incidental imaging findings suggesting Zinner syndrome in a young patient with pulmonary embolism: a case report. Radiol Case Rep 2020;15:437-41.

12. Florim S, Oliveira V, Rocha D. Zinner syndrome presenting with intermittent scrotal pain in a young man. Radiol Case Rep 2018;13:1224-7.

13. Naval-Baudin P, Carreno Garcia E, Sanchez Marquez A, Valcarcel Jose J, Romero NM. Multicystic seminal vesicle with ipsilateral renal agenesis: two cases of Zinner syndrome. Scand J Urol 2017;51:81-4.

14. Van den Ouden D, Blom JH, Bangma C, de Spiegeleer AH. Diagnosis and management of seminal vesicle cysts associated with ipsilateral renal agenesis: a pooled analysis of 52 cases. Eur Urol 1998;33:433-40.

15. Orhan I, Onur R, Cayan S, Koksal IT, Kadioglu A. Seminal vesicle sperm aspiration in the diagnosis of ejaculatory duct obstruction. BJU Int 1999;84:1050-3.

16. Cito G, Coccia ME, Sessa F, Cocci A, Verrienti P, Picone R, et al. Testicular fine-needle aspiration for sperm retrieval in azoospermia: a small step toward the technical standardization. World J Mens Health 2019;37:55-67.

17. Schlegel PN. Causes of azoospermia and their management. Reprod Fertil Dev 2004;16:561-72.

18. Cito G, Becatti M, Natali A, Fucci R, Picone R, Cocci A, et al. Redox status assessment in infertile patients with non-obstructive azoospermia undergoing testicular sperm extraction: a prospective study. Andrology 2020;8:364-71.

19. Xiang Y, Luo P, Cao Y, Yang ZW. Long-term effect of vasectomy on spermatogenesis in men: a morphometric study. Asian J Androl 2013;15:434-6.

20. Practice Committee of the American Society for Reproductive Medicine in collaboration with the Society for Male Reproduction and Urology. The management of obstructive azoospermia: a committee opinion. Fertil Steril 2019;111:873-80.

21. Ben Rhouma K, Marrakchi H, Khouja H, Attalah K, Ben Miled E, 
Sakly M. Outcome of intracytoplasmic injection of fresh and frozen-thawed testicular spermatozoa: a comparative study. J Reprod Med 2003;48:349-54.

22. Devroey P, Liu J, Nagy Z, Goossens A, Tournaye H, Camus M, et al. Pregnancies after testicular sperm extraction and intracytoplasmic sperm injection in non-obstructive azoospermia. Hum Reprod 1995; 10:1457-60.

23. Prins GS, Dolgina R, Studney P, Kaplan B, Ross L, Niederberger C. Quality of cryopreserved testicular sperm in patients with obstructive and nonobstructive azoospermia. J Urol 1999;161:15048.

24. Habermann H, Seo R, Cieslak J, Niederberger C, Prins GS, Ross L. In vitro fertilization outcomes after intracytoplasmic sperm injection with fresh or frozen-thawed testicular spermatozoa. Fertil Steril 2000;73:955-60.

25. Wald M, Ross LS, Prins GS, Cieslak-Janzen J, Wolf G, Niederberger CS. Analysis of outcomes of cryopreserved surgically retrieved sperm for IVF/ICSI. J Androl 2006;27:60-5.

26. Janzen N, Goldstein M, Schlegel PN, Palermo GD, Rosenwaks Z, Hariprashad J. Use of electively cryopreserved microsurgically aspirated epididymal sperm with IVF and intracytoplasmic sperm injection for obstructive azoospermia. Fertil Steril 2000;74:696701. 